Ciencia y Educación, Vol. 6, No. 1, enero-abril, 2022

ISSN (impreso): 2613-8794・ ISSN (en línea): 2613-8808

DOI: https://doi.org/10.22206/cyed.2022.v6i1.pp63-82

\title{
Formación del profesorado, enseńanza y evaluación. Experiencias de estudiantes portugueses
}

\author{
Teacher Education, Teaching and Assessment. \\ Portuguese Students' Experiences
}

\author{
Cláudia Pinheiro ${ }^{a}$ ORCID: 0000-0002-5474-8546 \\ Maria Assunção Flores ${ }^{\mathrm{b}}$ ORCID: 0000-0002-7483 \\ Francisco Cristóvão ${ }^{c}$ ORCID: 0000-0002-2191-9134
}

Recibido: 16/08/2021 • Aprobado: 16/11/2021

Cómo citar: Pinheiro, C., Flores, M. A., \& Cristóvão, F. Formación del profesorado, enseñanza y evaluación. Experiencias de estudiantes portugueses. Ciencia y Educación, 6(1), 63-82. https://doi.org/10.22206/cyed.2022.v6i1.pp63-82

\begin{abstract}
Resumen
Este artículo, procedente de un estudio más amplio, trata de comprender cómo los estudiantes que serán futuros profesores describen su experiencia en un curso de formación de profesores, concretamente en lo que respecta a las prácticas de enseñanza y evaluación. Los datos fueron recolectados a través de grupos focales con 30 estudiantes inscritos en un curso en el área de docencia. Los resultados apuntan a la prevalencia de prácticas docentes más tradicionales y a la falta de articulación entre la teoría y la práctica, aunque también hay aspectos que hacen referencia a diferentes grados de implicación de los alumnos en las actividades del aula y a diferentes dinámicas de interacción entre profesores y alumnos. Aunque los participantes destacan la importancia de la diversidad de los métodos de evaluación, y algunos admiten la relevancia de los portafolios y la retroalimentación oportuna y pertinente, sobresale el predominio de los exámenes y el trabajo en grupo.
\end{abstract}

Palabras clave: Educación Superior; estudiantes; evaluación; formación del profesorado.

\begin{abstract}
This article reports on findings from a wider study aimed at examining student teachers' experience in their teacher education program, as far as teaching and assessment practices are concerned. Data were collected through focus groups with 30 students enrolled in a program related to teaching. Findings point to the prevalence of more traditional teaching approaches and the lack of articulation between theory and practice, although aspects that refer to different levels of student involvement in classroom activities and different dynamics of teacher-student interaction were also identified. Although the participants emphasize the importance of the diversity of assessment methods, and some even admit the relevance of portfolios and timely and relevant feedback, tests and group work are the methods most used.
\end{abstract}

Keywords: Higher Education; students; assessment; teacher education.

\footnotetext{
a Universidade do Minho, Portugal. Correo-e: claudiampinheiro@hotmail.com

b Universidade do Minho, Portugal. Correo-e: aflores@ie.uminho.pt

C Universidade do Minho, Portugal. Correo-e: franciscojmcristovao@hotmail.com
} 


\section{Introducción}

La formación del profesorado es un tema central cuando se trata de mejorar la calidad de los profesores y de la enseñanza. Como señala Van Grieken (2016, p. 30):

Existe una plena conciencia de que la formación inicial docente constituye uno de los factores más relevantes de la calidad de la educación. Se espera que su impacto en el ejercicio profesional docente se evidencie en la dinámica de la escuela de hoy, que opera en un contexto definido por grandes cambios y transformaciones en todos los ámbitos de la sociedad, la ciencia y la cultura.

La formación inicial ha sido objeto de reestructuraciones más o menos profundas en todo el mundo, derivadas de diferentes motivaciones vinculadas al contexto político nacional e internacional (Valeeva \& Gafurov, 2017; Hammerness, van Tartwijk, \& Snoek, 2012; Mayer, Pecheone, \& Merino, 2012; Flores, 2011; Imig, Wiseman, \& Imig, 2011). Existe una diversidad de propuestas en cuanto a contenidos y organización, especialmente en lo que se refiere a la práctica docente (Flores, 2016) y diferentes formas de combinar los distintos elementos curriculares de los programas de formación (Flores, 2017).

En este sentido, es importante escuchar a los distintos participantes en la formación inicial sobre su filosofía y modo de funcionamiento, es decir, los alumnos que serán futuros profesores. En este artículo se presentan los datos de los estudiantes matriculados en un Máster en Enseñanza y en el Grado en Educación Básica. Nos centraremos específicamente en los desafíos, las prácticas de enseñanza, el aprendizaje y la evaluación.

\section{Formación del profesorado: teoría y práctica}

En los últimos años, la cuestión de la calidad de la educación y la formación del profesorado ha atraído la atención de muchos investigadores de todo el mundo
(Valeeva \& Gafurov, 2017; Maschke \& Stecher, 2016; Woolhouse \& Cochrane, 2015; Flores, 2011). La articulación entre la teoría y la práctica en la Formación Inicial del Profesorado (FIP) es un tema recurrente (Valeeva \& Gafurov, 2017; Flores, 2016; Van Nuland, 2011; Korthagen, Loughran, \& Russell, 2006), pero controvertido, siendo identificado como uno de los principales problemas que apuntan hacia una falta de conexión, más o menos evidente y explícita, entre una y otra (Flores, 2016). Aunque se reconoce que la práctica es un elemento central del currículo de la FIP, no hay consenso sobre sus objetivos, estrategias y competencias requeridas (Flores, 2016), aunque se reconoce la necesidad de apoyar la práctica en el aula y la investigación en el currículo de formación (Valeeva \& Gafurov, 2017) y la importancia de involucrar y escuchar a los futuros profesores.

Tang (2002), por ejemplo, destaca que los estudiantes que serán futuros profesores son agentes activos que emiten juicios evaluativos sobre las formas más teóricas de conocimiento aprendidas en la educación superior. Evans (2010), a su vez, concluyó que los futuros profesores se centran mucho en el aula y creen que solo la experiencia puede prepararlos realmente para la enseńanza. El estudio de Wæge \& Haugaløkken (2013) muestra la importancia directa de la teoría para las tareas diarias en el aula. Allen \& Wright (2014) también señalan que los estudiantes que serán futuros profesores valoran mucho los componentes teóricos y prácticos del curso. Cómo los estudiantes que serán futuros profesores dan sentido a su experiencia durante la formación inicial es un aspecto que es importante considerar para comprender sus experiencias subjetivas y evaluar los diferentes aspectos de la FIP (Tang, Wong, \& Cheng, 2016).

Las investigaciones que estudian la perspectiva de los estudiantes de magisterio sugieren que estos estudiantes tienden a priorizar la práctica sobre los aspectos más teóricos de la FIP (Mayer et al., 2015; Tang, Wong, \& Cheng, 2012) y a juzgar el valor de la teoría con respecto a su relevancia directa en las tareas cotidianas del aula (Tang et al., 2019; Wæge \& Haugaløkken, 2013). 
Uno de los elementos que ha recibido atención dentro de la formación inicial en varios países se relaciona con el componente de investigación como elemento clave en su desarrollo y mejora (Flores, 2016; Munthe \& Rogne, 2015). Flores (2016, p. 212) señala que este componente es inexistente en algunos programas, en otros casos no está explícito en el plan de estudios de la FIP, y corresponde a las instituciones de formación promover el desarrollo de habilidades de investigación, por ejemplo, durante las prácticas; pero en algunos contextos hay un componente curricular explícito sobre los métodos de investigación en el plan de estudios de la FIP que prosigue durante las prácticas. Se reconoce la importancia de que los estudiantes que serán futuros profesores desarrollen conocimientos y habilidades sobre la enseńanza y el aprendizaje a través de la investigación de su propia práctica, ya que aprender a ser profesor es un proceso complejo, contextual e idiosincrásico (Flores, 2019, 2006). Como sugiere Van Grieken (2016, p. 33), los estudiantes que serán futuros profesores tienen que:

Asumir la propia historia con una clara conciencia de la realidad; mantener una actitud no adaptativa, sino crítica frente a los problemas del ser humano y de la sociedad; asumir un compromiso con la transformación de su propio contexto. Se trata de un ser humano en permanente construcción y en constante diálogo con el contexto. Un sujeto situado cuya representación de la realidad nunca es estática, sino evolutiva. Por tanto, moviliza, con pasión, sus capacidades de reflexionar sobre ella, la interpela y la transforma.

De ahí la relevancia de la FIP de calidad con relación a cada contexto (Van Grieken, 2016) y en un momento histórico y político determinado. Para Van Grieken (2016, p. 39), hay que tener en cuenta varios aspectos:

- Una formación que parta de los intereses de los sujetos y de las especificidades del contexto;

- La superación de la visión estrictamente disciplinar enfocada en contenidos, considerando la visión inter y transdisciplinaria mediante proyectos o situaciones problematizadoras que permitan articular al aula la comunidad, la cultura, lo social, lo económico, lo político y lo emergente en la sociedad;

- Una formación crítica, propositiva y transformadora que promueva el ejercicio para la vida democrática y la construcción de ciudadanos y ciudadanas activos en el ejercicio de sus derechos y deberes;

- Una formación que construya identidades personales que permitan fortalecer la autoestima docente;

- Una formación que construya identidades nacionales, sociales y terrenales;

- Una formación que permita superar la tensión teoría-práctica y la conceptualice en una relación dialéctica, es decir, en la cual una dialoga con la otra y se integren como un todo;

- Una formación que, desde la complejidad, asuma la transitoriedad del conocimiento, su desconstrucción y reconstrucción permanente, pues no existen verdades acabadas;

- Una formación que en clave de competencia asuma como ejes la reflexión y la investigación-acción y que permita superar la concepción transmisiva del conocimiento, el aislamiento del trabajo docente y hacer de esta formación un espacio vivencial de problematización de los procesos de aprendizaje, generación de preguntas y propuestas de solución a las situaciones problemas que caracterizan la práctica;

- Una formación que articule la formación inicial con la formación continua durante todo el trayecto profesional docente;

- Una formación que integre los procesos formativos docentes con las TIC, la interculturalidad, la ética y los valores.

En el contexto portugués, y tras la implantación del proceso de Bolonia, todos los cursos de formación inicial del profesorado sufrieron cambios y se adoptó un modelo secuencial. Es decir, a partir de 2007, es necesario tener un Máster en Enseñanza (entre 90 y 120 ECTS) para acceder a la profesión docente, lo que ocurre después de una licenciatura de tres años. 
Así, en el caso de los educadores y profesores de ler ciclo (educación primaria), es necesario completar una Licenciatura en Educación Básica y luego acceder a una Maestría en Preescolar o 1er ciclo. Para la educación secundaria, por ejemplo, es necesario hacer un Máster en Enseñanza (Ej. en Matemáticas), después de haber obtenido una licenciatura que habilite en el área de la especialidad. Actualmente, el Decreto Ley vigente (Decreto Ley n. ${ }^{\circ}$ 79/2014) estipula cinco componentes del currículo de formación inicial: (i) área docente; (ii) área educativa general; (iii) didáctica específica (en las áreas de contenido y en la enseńanza de las materias del respectivo grupo docente); (iv) área cultural, social y ética; y (v) iniciación al ejercicio profesional.

\section{Perspectivas de enseñanza y evaluación: aportes para la formación del profesorado}

Roldão (2009) identificó tres matrices teóricas predominantes en las concepciones y prácticas docentes: (1) transmisible (basado en la transmisión de conocimientos); (2) constructivista (basado en la facilitación del aprendizaje); y (3) crítico (basado en la construcción activa y autónoma del aprendizaje). Por su parte, Trindade \& Cosme (2010), respecto a los paradigmas pedagógicos, distinguen tres paradigmas resultantes de la relación entre el profesor, el alumno y el conocimiento: (1) el paradigma de la educación (basado en la estrecha conexión entre el profesor y el conocimiento, la centralidad del profesor y la pasividad del alumno, y la memorización y las metodologías centradas en la adquisición de contenidos); (2) el paradigma del aprendizaje (basado en la estrecha conexión entre el alumno y el conocimiento, la centralidad de las estrategias de aprendizaje del alumno y las metodologías centradas en el alumno); y (3) el paradigma de la comunicación (en este paradigma el conocimiento se construye a través de la interacción de los profesores y de los alumnos, y está mediado por las experiencias de los interlocutores y el contexto). Se trata de dos importantes aportaciones a los enfoques de enseñanza y aprendizaje que pueden ser útiles para comprender las diferentes lógicas de la formación.

Una población estudiantil diversa y heterogénea requiere cambios en las prácticas de enseñanza, enfocándose menos en los enfoques centrados en el profesor y más en los resultados de aprendizaje que los estudiantes deben alcanzar (Brown, 2005; Miller, Imrie, \& Cox, 1998; Rust, 2002) en línea con una perspectiva más centrada en el alumno (Flores, 2019; Myers \& Myers, 2015; Webber, 2012; Black \& Wiliam, 1998). Diferentes razones (motivar a los alumnos, fomentar la actividad, proporcionar orientación y retroalimentación, calificar y seleccionar) repercutirán en la elección de los instrumentos de evaluación, que pueden incluir una amplia variedad de métodos adecuados para diferentes contextos y propósitos (Brown, 2005).

El papel central que desempeña la evaluación en el aprendizaje y en la enseñanza se reconoce cada vez más en el contexto de la educación superior (Hughes, 2011), porque la manera de evaluar a los alumnos puede influir en su forma de aprender (Brown, 2005), pero la evaluación tiene varios desafíos, generando tensiones y compromisos (Carless, 2007). Muchas prácticas de evaluación no promueven alumnos independientes, reflexivos y críticos, y este enfoque es incompatible con los objetivos académicos actuales (Freeman, 1995). En otras palabras, las presiones externas sobre la educación superior pueden hacer que la evaluación asuma una función principalmente sumativa.

Es importante entender cómo se ven las prácticas de evaluación, pero de forma constructiva y no solo desde una perspectiva de juicio (Brown, 2005). La retroalimentación formativa es muy importante, ya que debe ser detallada, exhaustiva, significativa, justa, estimulante y de apoyo, pero combinar todos estos aspectos puede ser una tarea difícil para los ocupados académicos/profesores (Brown, 2005). Para que el feedback sea efectivo (Ramon-Casas, 2019; Hughes, 2011) es necesario utilizar algunas estrategias sencillas que aumenten su impacto, sin aumentar la cantidad de feedback que se proporciona (Allal, 2020; Panadero, Jonsson, \& Strijbos, 2016). Así, proporcionando una retroalimentación adecuada y oportuna a 
los estudiantes (Gibbs, 1999), es posible mejorar los resultados del aprendizaje (Li \& Gao, 2016; Pope, 2001). Esto es aún más relevante en los cursos de formación de profesores, ya que los estudiantes que serán futuros profesores también necesitan aprender de la evaluación, pues serán profesores en el futuro y tendrán que evaluar a sus alumnos. Esto se refiere a la necesidad de explicitar las creencias y entender las prácticas como componentes de la enseñanza (Lutovac \& Flores, 2021a).

Diferentes autores se han dedicado a estudiar las concepciones de los profesores en formación sobre la evaluación, ya que se relacionan con el modo en que estos abordan la evaluación, así como el aprendizaje y el rendimiento de los estudiantes (Remesal, 2011; Brown \& Remesal, 2012; Flores et al., 2019; Lutovac $\&$ Flores, 2021a). Brown (2005), por ejemplo, identificó cuatro concepciones de la evaluación: (a) mejorar la enseñanza y el aprendizaje, (b) la responsabilidad de la escuela, (c) la responsabilidad del alumno y (d) la evaluación como algo irrelevante, mientras que Remesal (2011), basado en un estudio con 50 profesores, sostiene que las concepciones de la evaluación pueden, por un lado, estar relacionadas con la estructura del sistema educativo y pueden tender hacia concepciones más pedagógicas o más de responsabilidad que concierne a cuestiones externas sobre la escuela y, por otro lado, estar constituidas por diferentes creencias, a veces contrastantes, sobre el papel de la evaluación en la enseñanza y el aprendizaje considerados por separado. El mismo autor subraya que este hecho puede ayudar a comprender las dificultades asociadas a la aplicación de prácticas innovadoras de evaluación del aprendizaje.

Por su parte, Lutovac \& Flores (2021a), en un estudio realizado con 79 futuros profesores, identificaron cuatro concepciones de la evaluación: (1) la evaluación tiene que ver con la retroalimentación y la reflexión, (2) la evaluación debe ser personalizada y demostrar el aprendizaje de los estudiantes, (3) la evaluación debe tener en cuenta el esfuerzo de los estudiantes y, por último, (4) la evaluación no permite medir el éxito o el fracaso de los estudiantes. Estos autores concluyeron que el profesor desempeña un papel determinante en el fracaso de los alumnos, del que debe aprender para reformular sus prácticas de enseñanza y/o evaluación, destacando la preponderancia de la evaluación formativa, así como la necesidad de hablar con los alumnos y proporcionarles retroalimentación. En general, la primera concepción se basa en la idea de que la evaluación sirve para mejorar y, por tanto, puede ayudar a los alumnos y a los profesores a aprender. Las dos concepciones siguientes reflejan la idea general de que la evaluación debe ser un proceso continuo y tener en cuenta los antecedentes y la trayectoria de los estudiantes. Finalmente, la cuarta concepción de evaluación identificada se relaciona con el hecho de que las calificaciones y los exámenes no permiten medir los propósitos morales y sociales del aprendizaje y con la subjetividad en la interpretación del fracaso en el contexto de la evaluación.

Este estudio presenta las percepciones de los estudiantes que serán futuros docentes sobre los desafíos en la educación superior, las prácticas docentes, los factores que influyen en su aprendizaje y cómo son evaluados.

\section{Metodología}

Este estudio forma parte de un proyecto de investigación más amplio titulado The use of alternative methods of assessment in higher education: $A$ study of university teachers and students, financiado por la Fundação para a Ciência e Tecnologia (SFRH/ BD/122094/2016). El estudio fue aprobado por el Comité de Ética para la Investigación en Ciencias Sociales y Humanidades de la Universidade do Minho (Ref. SECSH 037/2016).

Este estudio tuvo como principales objetivos: i) comprender los desafíos que enfrentan los estudiantes que serán futuros docentes en la educación superior; ii) conocer cómo perciben las prácticas docentes los futuros docentes; iii) comprender los factores que influyen en su aprendizaje; iv) conocer las percepciones de los futuros profesores sobre la evaluación. 
Los datos se recolectaron en una universidad pública portuguesa de enero a julio de 2018, a través de 5 grupos focales (véase el Cuadro 1). Se invitó a los grupos de discusión a los estudiantes que asistían a un curso de formación de profesores y 30 participantes se ofrecieron como voluntarios. Como pretendíamos conocer las percepciones de los estudiantes que se convertirán en futuros profesores, optamos por realizar grupos focales, ya que permiten una representación en la que se refleja la dinámica de una realidad y se centra en un tema concreto, explorándolo en profundidad, destacando el cuestionamiento en torno a un determinado tema o tópico, la interacción grupal y la construcción conjunta de significados. Esta opción se justifica por ser un método en el que los participantes se sienten cómodos para dar su opinión sobre el tema analizado, en un contexto en el que el discurso fluye de forma natural hacia la comprensión de lo que la gente realmente siente y piensa (Krueger \& Casey, 2009). El investigador se interesa por la forma en que los individuos interactúan como miembros de un grupo, discutiendo el mismo tema. Lo importante es analizar cómo responde cada persona a los demás participantes para construir una perspectiva analítica a partir de la interacción en el grupo.

En los grupos focales participaron 30 estudiantes, 23 de la Maestría en Docencia y 7 de la Licenciatura en Educación Básica (véase el Cuadro 1). De estos participantes, 5 eran hombres y 25 mujeres, con edades comprendidas entre los 20 y los 40 años. Los alumnos de la Licenciatura en Educación Básica fueron incluidos en este estudio porque para ser educador (preescolar) y profesor del 1er ciclo (primaria) es necesario realizar un máster posterior a esta licenciatura. En el caso de los demás másteres, los alumnos proceden de varias licenciaturas.

\section{Cuadro 1}

Número de participantes por grupo de discusión

\section{Ciclo de estudios Identificación del grupo
focal Número de participantes
por grupo de discusión}

\begin{tabular}{|c|c|c|c|}
\hline \multirow{4}{*}{ Maestría en Docencia } & GF1 & 6 & $40 \mathrm{~min}$. \\
\hline & GF 2 & 3 & $61 \min$. \\
\hline & GF 3 & 7 & $44 \mathrm{~min}$. \\
\hline & GF 4 & 7 & $50 \mathrm{~min}$. \\
\hline $\begin{array}{l}\text { Licenciatura en Educación } \\
\text { Básica }\end{array}$ & GF 5 & 7 & $62 \mathrm{~min}$ \\
\hline Total & & 30 & 4h 30min. \\
\hline
\end{tabular}


Las intervenciones de los participantes fueron grabadas en audio (con autorización previa), con un total de cuatro horas y treinta minutos de conversación. Posteriormente, las intervenciones de los participantes fueron transcritas en su totalidad y sometidas a un análisis de contenido, respetando todos los principios éticos en un proceso de investigación de esta naturaleza. Para el análisis de los discursos pronunciados, utilizaremos la técnica de análisis de contenido (Bardin, 2013), ya que ayuda al investigador a mantenerse alejado de las interpretaciones espontáneas, basándose en “criterios que se centran más en la organización interna del discurso que en su contenido explícito" (Quivy \& Campenhoudt, 2003, p. 228). Realizamos el análisis del discurso de los grupos focales privilegiando un análisis temático inductivo a partir del cual buscamos identificar los principales ejes de lectura de los datos, teniendo en cuenta los principios y lineamientos de la literatura en esta área (cf. por ejemplo, Bryman, 1988; Silverman, 2000).

El guion del grupo de discusión comprendía cuatro dimensiones que se presentan en el Cuadro 2, así como ejemplos de las preguntas formuladas.

\section{Cuadro 2}

Dimensiones de los grupos focales y ejemplos de preguntas formuladas

\section{Dimensiones del grupo focal \\ Cuestiones}

\section{i) Ser estudiante de educación superior}

- ¿Cuáles son los principales retos como estudiante de educación superior?

- Teniendo en cuenta su experiencia como estudiante universitario, ¿cuáles son las mayores dificultades a las que se enfrenta actualmente? ¿Cómo intenta superarlas? ii) El proceso de enseńanza y aprendizaje
- ¿Cuáles actividades son las que más realiza en el contexto del aula?

- ¿Cuáles métodos o estrategias de enseñanza son los más utilizados por los profesores? Poner ejemplos.

- ¿Cuáles aspectos influyen positiva y/o negativamente en su aprendizaje? ¿Por qué?

- ¿Cuáles son los métodos de evaluación más utilizados para valorar su

iii) Evaluación aprendizaje?

- ¿En qué medida influyen los métodos de evaluación utilizados en su forma de estudiar? Poner ejemplos.

$\begin{array}{lll}\text { iv) Relación evaluación/ } & \text { ¿ ¿Cómo influye la evaluación en su aprendizaje? } \\ \text { aprendizaje } & \text { - ¿En qué medida la evaluación puede mejorar el aprendizaje? }\end{array}$ 
Los resultados se presentan según las categorías emergentes que surgen del análisis de los datos: a) desafíos como estudiantes universitarios; b) prácticas de enseñanza; c) factores que influyen en el aprendizaje; y d) evaluación, como se comprueba en la Figura 1 presentada a continuación.

\section{Figura 1}

Categorías de análisis de los discursos de los estudiantes

\section{Desafíos}

- Mayor carga de trabajo

- Diversidad de tareas y solicitudes

-Gestión de actividades

- Falta de apoyo de los profesores

-Transición de la enseñanza secundaria a la superior

- Dificultades en el uso de la lengua inglesa

-Conciliación de la vida personal y profesional con la vida académica

\section{Prácticas pedagógicas}

- Prevalencia de las prácticas centradas en el profesor

-Predominio de las clases expositivas y el uso de PowerPoint

- Falta de articulación con la práctica profesional

-Falta de coherencia entre el discurso y la práctica

\section{Factores que influyen en el aprendizaje}

- Número de estudiantes por clase

-Dinámica/interacción en el aula

- Nivel de participación de los estudiantes en las actividades del aula

- Clima de trabajo en el aula

-Enfoque dialógico

-Actitud del docente en el aula

-Relación con los docentes

\section{Evaluación}

- Predominio de los exámenes y del trabajo en grupo

- Aspectos negativos de los exámenes y del trabajo en grupo

-(In)justicia en la calificación de las presentaciones orales de los trabajos

-Predominio de una actividad clasificatoria

-Valorización de los métodos alternativos de evaluación

- Complejidad del acto de evaluación

- Ausencia de retroalimentación periódica y sistemática

- La retroalimentación como una oportunidad para mejorar el aprendizaje 


\section{Resultados}

En esta sección presentamos los datos derivados del análisis de los grupos focales realizados con estudiantes que serán futuros profesores. Los datos referidos siguen la categorización emergente identificada en la sección anterior.

\section{Desafíos encontrados}

Los retos que señalan los estudiantes de la enseñanza superior están asociados a una serie de factores, que están directamente relacionados con el proceso de enseńanza y aprendizaje, pero también con la creciente carga de trabajo y la falta de apoyo de los profesores.

En cuanto a los desafíos a los que se enfrentan como estudiantes en la enseñanza superior, se mencionó el tema de la fragmentación curricular en los cursos de formación docente que también se discute en otros contextos (Flores, 2016), y que se deriva de la falta de articulación entre las diferentes unidades curriculares y los contenidos a lo largo del curso, lo que remite al aumento de la carga de trabajo: Los dos últimos cuatrimestres fueron muy agotadores en cuanto a trabajos a entregar, con plazos muy ajustados, era difícil gestionar eso (E9). Otros aspectos se refieren a la diversidad de tareas y exigencias a las que tienen que hacer frente: Teniamos muchas asignaturas y muy diferentes, cada disciplina requería un trabajo exhaustivo, teníamos tareas, exámenes, teníamos tareas cada semana. (E8). La gestión de las actividades a realizar también fue señalada por los alumnos que serán futuros profesores como un desafío al que tienen que enfrentarse y que se deriva del volumen de trabajo, pero también de la naturaleza de las tareas a realizar, concretamente en lo que respecta a los momentos de evaluación:

Cuando hablamos de tener más trabajo, hay profesores que piden un trabajo, un examen, luego es mucho trabajo. El trabajo requiere compromiso, pero si se trata de un trabajo individual y en grupo, ¡vale! $A$ veces hay más de dos tareas por asignatura y no tenemos tiempo. (E6)

Destacamos la falta de apoyo por parte de los profesores y la ausencia de aclaraciones más explí- citas que son más evidentes en el momento de acceder a la educación superior. Este aspecto puede estar asociado a la especificidad del modelo de enseńanza secundaria en el contexto portugués en lo que respecta, en particular, a las cuestiones de evaluación y a la naturaleza del trabajo pedagógico:

En las tareas que se proponen y en los exámenes, cuando entramos aquí [en la universidad], todo es muy diferente, quizás necesitamos más aclaraciones de los profesores para conocernos mejor. (E5) Tenemos otras cosas que hacer. Ellos [los profesores] ponen las cosas el mismo día en la plataforma y nosotros no podemos, tenemos otras cosas que hacer. (E13)

Esta opinión es corroborada por los alumnos de la licenciatura, que destacan la diferencia que se siente en el paso de la enseńanza secundaria a la superior, ya que es algo que todavía está muy presente debido a la proximidad del tiempo, principalmente en lo que se refiere a la falta de seguimiento por parte de los profesores y a la forma de organizar la información y los contenidos del plan de estudios:

No tener tanta ayuda del profesor. En el instituto de enseñanza secundaria tenemos empatía con los profesores, hay una relación. Aqui, en la enseñanza superior, no tenemos tanto... también porque somos más estudiantes, porque el grupo tiene más alumnos. Porque hay más [alumnos] dentro del aula. (E2)

Mientras que otros años teníamos un manual, cuando llegamos aqui tenemos que organizar el contenido nosotros mismos y conseguirlo por iniciativa propia. (...) Es una carga enorme. (E12)

Los participantes también mencionaron las dificultades para utilizar la lengua inglesa, ya que, aunque los alumnos a lo largo de su trayectoria escolar cursan la asignatura de inglés, identifican dificultades asociadas a su nivel de competencia:

Especialmente cuando los profesores nos dan tareas para leer y comprender el idioma, que es complejo. (E13) 
El problema es cuando está en otro idioma. Todo el mundo cree que sabemos inglés, pero hay mucha gente que no lo sabe y leer en inglés es muy complicado. (E15)

En los últimos años, debido a los cambios que se han producido en las formas de acceso a la educación superior, creando oportunidades y condiciones para que aquellos que habían abandonado la educación hace unos años pudieran entrar también para obtener una formación académica superior, como es el caso, por ejemplo, del acceso de los mayores de 23 años, o incluso por la necesidad económica emergente con mayor expresión desde hace unos años, más estudiantes son trabajadores, de ahí que se hable de la conciliación de la vida personal y profesional con la académica. También hay una reflexión sobre la motivación para aprender y estudiar:

El mayor desafio fue el tiempo y luego ser estudiante-trabajador; también fue difícil de conciliar, pero creo que los profesores también tienen en cuenta nuestra opinión en términos de evaluación, (E10)

En mi caso, es difícil conciliar el trabajo y la Universidad, es agotador. Han sido semanas muy agotadoras. (E29)

\section{Prácticas pedagógicas}

Como futuros profesores, además de prestar atención a los contenidos que se les enseñan, también valoran la forma en que se les transmiten los conocimientos, porque esperan que se les enseñe a enseñar. De sus discursos sobresale el predominio de las prácticas centradas en el profesorado, que asocian con cierta incoherencia entre el discurso y la práctica y la asunción de un papel pasivo por parte de los alumnos:

Se nos dice que no debemos seguir la enseñanza tradicional, pero las prácticas que adoptan [los profesores] son de enseñanza tradicional. Los alumnos acaban teniendo una actitud pasiva. (E5)

Luego también tenemos el problema de no interrumpir, de interrogar. No hay tanta apertura. Nos volvemos más retraidos. (E3)
Pasivamente, acabamos aceptando. Hay diálogo, es participativo, pero también como estudiantes, siempre dependemos de algo que se nos propone, y a partir de ahi nos expresaremos. (E9)

Los datos también señalan el predominio de las clases expositivas y el uso de PowerPoint, que acaba siendo repetitivo y poco estimulante, lo que conduce a una actitud más pasiva por parte de los alumnos. Este aspecto se refiere al papel de los formadores de profesores, cuyas prácticas pedagógicas influyen de manera decisiva en el proceso de formación de los futuros profesores, especialmente en lo que se refiere a la explicitación de la pedagogía y la clarificación de los propósitos y las acciones como estrategia que promueve la co-construcción del conocimiento profesional.

Lectura en PowerPoint. La mayoría utiliza el PowerPoint, pero lee lo que hay, no lo explica en otras palabras, nosotros sabemos leer el PowerPoint. (E2)

En mi opinión, el curso debería ser más práctico... Hablo en general, pero llegar a clase, abrir el PowerPoint y empezar a leer, hablar de toda la teoría.. la clase ha terminado. Algunas clases son asi. Hay clases que no deberian ser tan teóricas. (E11)

Escuchar al profesor [risas] y pequeños debates. Creo que hablo en nombre de todos cuando digo que tenemos muchas clases expositivas. (E24)

$\mathrm{Al}$ ingresar a un curso de formación docente, los estudiantes esperaban una mayor articulación con la práctica profesional, mencionando la falta de coherencia entre el discurso y la práctica que identifican en los formadores de docentes, señalando incluso la disonancia entre la pedagogía que defienden a nivel discursivo y su contradicción en la práctica en el aula:

Nuestras clases deberian ser más prácticas. El curso tiene que ser más práctico, porque terminamos y si entramos en un aula con estudiantes, no sabemos qué hacer. (E11)

Nuestra profesión es muy práctica y voy al contexto sin saber cómo actuar, cómo hacer, tenemos estos temas teóricos y por lo tanto menos práctica (E6) 
No sabemos cómo pasar de la teoría a la práctica, necesitamos apoyarnos en la teoría, pero nos falta mucha práctica. (E4)

No aprendemos a enseñar (...). Por ejemplo, no sé cómo enseño a aprender a leer. Esa teoría es necesaria, pero cómo aprenden a escribir, cómo aprenden a leer. (E6)

Decimos que el estudiante aprende a través de la acción, pero sabemos que asi funciona con todos los seres humanos. (E5)

Se nos dice que debemos utilizar un método más didáctico para enseñar a los estudiantes, pero luego ellos [los profesores] hacen lo contrario con nosotros. (E36)

Los que enseñan pedagogía son los que menos aplican estas cosas en la práctica, dan lecciones más expositivas. (E32)

\section{Factores que influyen en el aprendizaje}

El número de alumnos por clase surgió como uno de los factores que influyen en el aprendizaje. Una clase con demasiados alumnos puede condicionar el aprendizaje, por un lado, en el tipo de lecciones que se impartirán, en la organización del aula y de los tiempos de enseñanza y, por otro lado, en la intervención que realizará el alumno que estará más condicionada a un momento más corto, para dar la oportunidad de que todos participen y expongan sus dudas y perspectivas; pero, por otra parte, no hay diversidad de opiniones y perspectivas que podrían enriquecer el aprendizaje:

El hecho de que haya pocos estudiantes en este máster ha sido beneficioso para nosotros. Las orientaciones, la atención se centra más en nosotros. Por otro lado, hay menos puesta en común, opiniones, perspectivas, pero en algunas asignaturas en las que ya hemos estado compartiendo clases con compañeros estudiantes de otros másteres, el aula tenía un mayor número de alumnos, era un poco más difícil de gestionar en cuanto a fechas de evaluación, de hacer los trabajos, porque era dificil gestionar esa parte, porque perdiamos mucho tiempo. (E8)

La dinámica/ interacción en el aula y el enfoque dialógico aparecen como factores que influyen en el aprendizaje, marcando el ambiente del aula y el grado de implicación de los alumnos en las actividades del aula:

La dinámica que tenemos con los profesores también es importante, para facilitar nuestro aprendizaje y también para entender nuestra parte, que a lo largo del semestre siempre tuvimos ese entendimiento de los profesores. (E10)

Tenemos que involucrarnos en el tema en lugar de que sea algo muy expositivo. (E17)

Tener interacción con el profesor y con los contenidos, eso es muy bueno. Tenemos que luchar contra las clases más expositivas. Aprender a enseñar para que el alumno pueda crear conocimiento y construir su propio razonamiento. (E25)

Una vez más, los participantes señalaron la discrepancia entre lo que el profesor hace y lo que defiende: Los profesores defienden una cosa y cuando se trata de aplicarla con nosotros, no lo hacen. (...) la profesora no aplicó lo que defendia (E31), especialmente en lo que respecta a cuestiones de comunicación y actuación en el aula: Un lenguaje complejo, me pierdo en las ideas. El profesor habla de lo que debe hacerse y hace lo contrario (E11). La actitud del profesor en el aula surgió como un factor que influye en el aprendizaje, ya que puede afectar al interés de los alumnos por la asignatura, los contenidos o incluso los propios alumnos. De nuevo, se destaca la alusión al papel de los formadores de profesores y cómo pueden moldear la práctica docente de los futuros profesores en el sentido de que la formación del profesorado es mucho más que un entrenamiento de habilidades, refiriéndose a "un proceso educativo que permite el desarrollo de profesionales críticos, informados y altamente competentes" (Loughran, Keast, \& Cooper, 2016, p. 416):

El comportamiento de los profesores hacia nosotros en el aula. Son cuatro horas de clase devaluando [la Licenciaturalel Máster] y a los alumnos. ¿Cuál es la motivación para trabajar? (E14)

Hay clases en las que me apetece venir aqui la la universidad], aunque tenga sueño, sé que me voy a 
despertar, otras, en las que pienso: “Para qué me voy a levantar de la cama? (E13)

Me interesa mucho más la asignatura cuando el profesor muestra pasión por lo que hace, cosa que noto, porque vamos a ser profesores. No sé si hay profesores a los que no les gusta enseñar, pero hay claras diferencias entre los profesores. (E31)

Por último, la relación con los profesores también es identificada por los estudiantes que serán futuros profesores como un factor que influye en su aprendizaje porque, en su opinión, es un factor decisivo para la calidad de la enseñanza y el éxito del aprendizaje.

Creo que siempre tuvimos una buena relación con nuestros profesores. (E10)

Luego hay otros con los que nuestra relación es tan problemática que nos hace olvidar los buenos. (E11)

\section{Evaluación}

En la dimensión de la evaluación, los alumnos que serán futuros profesores destacaron la prevalencia de los exámenes y del trabajo en grupo, tendiendo a valorar este último por las oportunidades que ofrece en términos de interacción y aprendizaje:

Exámenes. Hemos tenido portafolios, reflexiones, pero sobre todo es una tarea y un examen o dos exámenes. En mi opinión, me gusta mucho hacer trabajos en grupo, porque creo que nos enriquece más escuchar la opinión de los compañeros (...) con los compañeros investigamos más, nos repartimos el trabajo y además es menos pesado para nosotros. Asi que, a nivel de aula, también creo que el trabajo en grupo es positivo, porque los otros grupos nos pueden cuestionar y vemos si el trabajo está bien hecho, qué podemos hacer mejor. (E6)

Trabajo mayoritariamente en grupo, con presentación. (E33)

Los estudiantes mencionan el examen como uno de los métodos de evaluación más utilizados por los profesores, reconociendo los aspectos negativos que tiene respecto a la calidad del aprendizaje y su importancia, pero también lo que realmente representa en cuanto a los resultados obtenidos, cuando es el momento de hacer un balance del aprendizaje realizado, cuestionando su eficacia y adecuación.

El examen es el resultado de ese momento. El examen nos limita mucho. (E4)

Creo que los exámenes nos limitan mucho, porque se puede hacer en un día dificil. No haber estudiado muy bien, no entender las preguntas. Acabamos siendo evaluados, imaginemos que son dos pruebas, sale mal, se nos evalúa por ello. (E3)

En un examen, puedes saber cómo copiar mejor que los demás. (E14)

No evalúa los conocimientos, sino la interpretación que hacemos de la pregunta. No pregunta las cosas con objetividad. (E18)

En el examen hay más presión. No muestra los conocimientos que el alumno tiene o no tiene. No aprendemos mucho si la evaluación se limita a un examen, porque si aprendemos a lo largo del semestre, japrendemos mejor! (E26)

Cuando se trata de los exámenes, es sólo lo que hay, nada más. Lo que haces ahi [en el examen], lo que escribes es lo que se evalúa. (E14)

Los estudiantes también se refirieron a las situaciones de estrés asociadas a los exámenes y a la demostración real de lo que saben en un momento determinado. Sin embargo, también identificaron aspectos negativos del trabajo en grupo, especialmente cuando no funciona bien, cuando no hay una buena gestión del tiempo y de las tareas:

El trabajo en grupo funciona muy bien cuando todos quieren trabajar, pero siempre hay dos o tres a los que no les interesa, estoy cansado de arrastrar a la gente. (E14)

El trabajo siempre se acaba haciendo, pero hay gente que sabe que está en ese grupo y ya sabe que los demás van a trabajar y les da igual. (E11) 
Otros se refieren a la calificación y a la justicia (o injusticia) cuando la contribución de los distintos miembros del grupo no es equivalente y cuando se producen presentaciones orales de los trabajos. Este aspecto puede estar asociado a las dificultades en la gestión del tiempo y de las tareas, haciendo referencia a la importancia de las habilidades de autorregulación, pero también a las estrategias de seguimiento y evaluación del trabajo realizado:

Puesto que la nota del trabajo es igual, entonces hay una presentación para diferenciar a los estudiantes. (...) porque hay gente que no trabaja o no quiere saber. (E6)

Pero eso es un poco injusto [diferenciar la nota de la presentación], porque a veces la gente que no hace el trabajo lo hace mejor en la presentación que los que tienen más conocimientos sobre el tema. Eso tiene que ver con la personalidad de la persona. Por eso es incluso lógico que el trabajo en grupo no sea el unico método de evaluación. (E5)

Tenemos que saber trabajar con todo tipo de personas, en diferentes momentos, pero el problema es nuestra evaluación. En el trabajo en grupo, una persona se queda con todo el trabajo y la otra recibe la misma nota que la que lo ha hecho todo. (E11)

Otro aspecto que se tuvo en cuenta en la dimensión relacionada con la evaluación fue el significado de las calificaciones/resultados, que, en su opinión, la mayoría de las veces no reflejan los conocimientos que el alumno sabe o ha aprendido. Este aspecto se debe a que el alumno es evaluado, la mayoría de las veces, de forma sumativa con relación a la cual los alumnos tienen una perspectiva crítica, cuestionando la pertinencia y adecuación de los métodos y criterios de evaluación:

Las calificaciones no dicen nada de lo que sabemos. (E13)

Los resultados no demuestran lo que sabemos. No sé nada de lo que ya he estudiado, pero ya está hecho. (E11)

Creo que es relativo. No sé de qué soy capaz. No sé si la nota que tengo refleja lo que sé. (E28)
A veces sé más de lo que hay, pero a veces ocurre lo contrario. (E24)

En cuanto a las calificaciones que obtengo, tal vez ni siquiera trabajé y veo que mis compañeros también obtuvieron buenas calificaciones, tal vez piensan como yo. (E24)

Como la formación es muy teórica, tener una media de 18 al finalizar el máster no significa que yo sepa enseñar. (E32)

El concepto de evaluación como clasificación surgió de la lectura de los datos recolectados: La evaluación está muy centrada en la clasificación, no se preocupan por la calidad, sino por la cantidad. (E26), que se remite a una lógica más cuantitativa y centrada en los resultados.

A pesar de su uso residual, los alumnos que serán futuros profesores valoraron los métodos de evaluación alternativos, admitiendo que permitían una mayor implicación de los alumnos, permitían la aplicación de lo aprendido y posibilitaban la construcción del conocimiento basada en el aprendizaje y el trabajo continuo, con autorreflexión y autoevaluación:

Con estos métodos [alternativos], nos implicamos más. (E11)

Se activa inconscientemente lo que se aprende, sin tener que memorizarlo. (E14)

Cuando se evalúa en cada clase, se puede ver quién está trabajando o no, y es una forma de que el profesor entienda lo que se ha hecho alli. Se hizo en clase, presentamos en clase, trabajamos en clase, todos estamos obligados a trabajar en esa clase. (E11) El portafolio es algo más libre y podemos expresar nuestros conocimientos, nuestra opinión, en el examen no podemos hacerlo. Es más riguroso. Se adapta más a lo que exige el profesor y no tanto a la autorreflexión. No tenemos esa libertad. En muchos exámenes no éramos nosotros los que pensábamos, sino los que memorizábamos. En el examen nos preparamos para ese momento, a partir de ahi aprobamos, desconectamos, 
lo guardamos en una caja y ya está. En el trabajo, siempre pienso en lo que se podría mejorar o si estaba bien. (E24)

Aprendemos más si se trata de una tarea o un portafolio, porque construimos cosas. (E27)

Un trabajo en grupo nos exige más investigación, más reflexión, preparación. También estamos construyendo nuestro conocimiento. (E7)

Los participantes en el estudio también reconocieron la complejidad del acto de evaluación, sugiriendo que: La evaluación es realmente muy compleja, no sé si es peor evaluar o ser evaluado, influyen muchos factores, se necesitan otros métodos. (E26). Por lo tanto, es necesario situar la evaluación en su amplitud y complejidad, incluyendo su dimensión política y ética. Son aspectos que deben ser discutidos en el contexto de la formación del profesorado para superar una visión más tecnicista, que implica la explicitación de creencias y prácticas y la discusión de las implicaciones de las opciones tomadas en el ámbito de la evaluación sobre la calidad de la enseñanza y el aprendizaje.

También en la dimensión de la evaluación, los estudiantes que serán futuros profesores mencionaron el aspecto relacionado con la ausencia de una retroalimentación sistemática y regular por parte de los diferentes profesores, afirmando que solo tienen retroalimentación a través de las notas que reciben por los exámenes, trabajos o incluso al final del semestre:

Hubo un colega que le pidió al profesor que le diera su opinión y ni siquiera le contestó. (E10)

No recibimos información, sólo al final del semestre. (E11)

En un examen, recibimos la nota y no sabemos qué hacer para mejorar. (E24)

Entonces, también nos sentimos intimidados, porque si el profesor no nos transmite el feedback, aún no ha salido la nota final y vamos a pedir justificaciones, no sabemos cómo lo interpreta. Podría ofenderse y bajarme la nota. (E33)

No siempre recibimos información a tiempo, no estoy seguro de si estoy haciendo las cosas bien o no. No hay respuesta. (E14)

A pesar de no recibir información de forma regular y sistemática, los participantes en el estudio reconocieron el feedback como una oportunidad para mejorar el aprendizaje, lo que de nuevo apunta a cuestiones de seguimiento y balance de las estrategias utilizadas, remitiendo la evaluación únicamente al momento de otorgar las calificaciones:

Si, definitivamente si. Para saber en qué nos equivocamos, para entender, para ser mejores en el futuro. (E6)

Incluso para que podamos reflexionar sobre lo que hacemos. También necesitamos tener esa competencia para ser mejores profesores y educadores. (E7)

La Figura 2 resume el análisis de los datos cualitativos recogidos de los participantes. Si bien los retos a los que se enfrenta la enseñanza superior están relacionados con el aumento de la carga de trabajo y la falta de apoyo de los profesores, también suscitan una reflexión sobre cuestiones más amplias de la vida académica, como la transición de la enseñanza secundaria a la superior, sobre cuestiones personales (conciliación de la vida personal con los estudios de enseńanza superior) y sobre cuestiones pedagógicas, concretamente los métodos de enseńanza y evaluación y la forma en que los estudiantes aprenden a ser profesores en el contexto de un curso de formación inicial del profesorado. Esta reflexión sobre cómo se enseña y cómo se cree que se va a enseñar es fundamental en el proceso de convertirse en profesor, permitiéndole desvelar y cuestionar creencias y teorías implícitas o reforzarlas, así como desarrollar una perspectiva más sostenida y crítica sobre el proceso formativo, especialmente en lo que respecta a la pedagogía y la evaluación. 


\section{Figura 2}

Resumen de los datos cualitativos que surgen de los discursos de los participantes
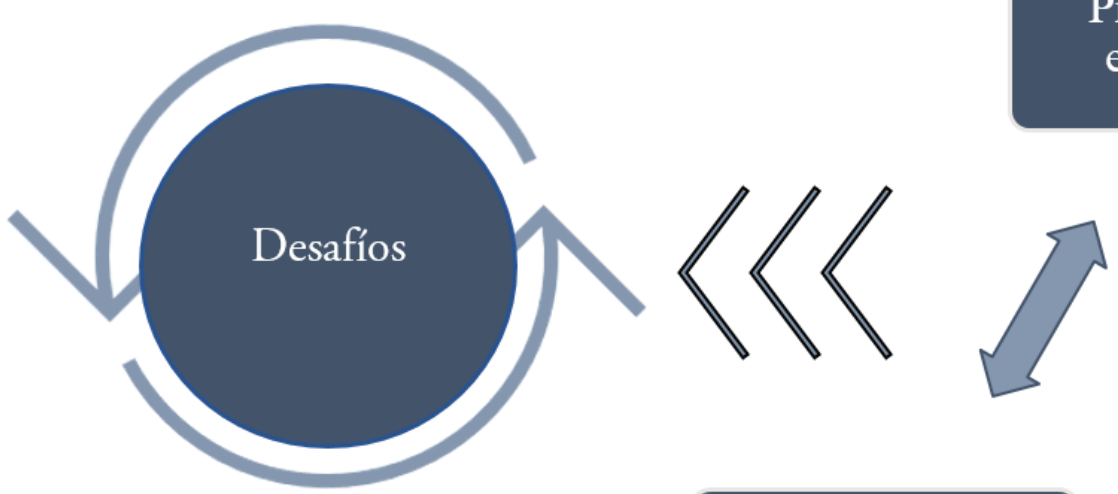

Prácticas de

enseñanza

Factores que

influyen en el

aprendizaje

Evaluación

\section{Discusión y conclusiones}

Este estudio aporta una visión cualitativa que amplía el conocimiento sobre las percepciones de los futuros profesores sobre aspectos que les resultan imperiosos como futuros profesores, especialmente en el ámbito de la evaluación. Identificamos cuatro dimensiones extraídas de los discursos de los participantes: a) desafíos; b) prácticas de enseñanza; c) factores que influyen en el aprendizaje; y d) evaluación.

La primera dimensión, relativa a los retos a los que se enfrentan los estudiantes universitarios, reúne aspectos que permiten comprender la entrada de los estudiantes y futuros profesores en la enseñanza superior desde otra perspectiva, teniendo en cuenta la transición gradual y compleja de estudiante a profesor. Todos los aspectos destacados se deben a las exigencias impuestas en la cambiante sociedad actual, a la necesidad de responder a varias demandas simultáneamente y en poco tiempo a tener una formación especializada, realizando todas las tareas con la máxima eficacia y eficiencia, lo que puede crear una enorme presión en la vida personal, profesional y académica, ya que las diferentes situaciones y los distintos entornos que los estudiantes, en la fase de adaptación a la Educación Superior, experimentan pueden tener implicaciones en las decisiones de permanencia y abandono de los cursos (Nunes \& Garcia, 2010; Almeida \& Cruz, 2010) o en la gestión de las expectativas respecto al curso, influyendo en su motivación y/o interés por este.

Por su parte, las dimensiones relacionadas con las prácticas docentes, los factores que influyen en el aprendizaje y la evaluación corroboran, por un lado, el estudio de Remesal (2011), que destaca la importancia de la evaluación en el aula, y el estudio de Lutovac \& Flores (2021a), respecto a las concepciones de la evaluación, especialmente las asociadas a la medición del éxito o el fracaso de los estudiantes $y$, en cambio, su relación con la retroalimentación y la reflexión sobre el proceso de aprendizaje, lo que sugiere la necesidad de considerar las experiencias de los estudiantes y la visión del fracaso desde la perspectiva de los estudiantes pero también de los (futuros) profesores (Lutovac \& Flores, 2021b).

En cuanto a las prácticas de enseñanza, los participantes mencionaron estar más centrados en el profesor, observando un paradigma de instrucción 
directa, donde se establece un límite al que puede llegar el alumno, tal como preconizan Barr \& Tagg (1995), en lugar de un paradigma de aprendizaje, en el que el éxito marca su límite (Barr \& Tagg, 1995), adoptando prácticas más centradas en el alumno (Flores et al., 2019; Myers \& Myers, 2015; Webber, 2012). Los datos recogidos apuntan al predominio de las prácticas docentes basadas en la matriz transmisible -basada en la transmisión de conocimientos (Roldão, 2009) y un paradigma pedagógico de la educación basado en la estrecha conexión entre el profesor y el conocimiento, la centralidad del profesor y la pasividad del alumno, y la memorización y las metodologías dirigidas a la adquisición de contenidos (Trindade \& Cosme, 2010). También se refieren a la falta de articulación con la práctica profesional, a pesar de que los discursos internacionales señalan la importancia de la práctica en los cursos de formación docente (Mayer et al., 2015; Tang, Wong, \& Cheng, 2012), sin dejar de lado la relevancia de la teoría en el contexto diario del trabajo en el aula (Wæge \& Haugaløkken, 2013; Tang, Wong, \& Cheng, 2016).

En cuanto a los factores que influyen en el aprendizaje, estos se presentan principalmente como una fuente de restricción para la construcción del conocimiento por parte del alumno, condicionando su forma de estar dentro del aula y su intervención/ participación. Las circunstancias en las que el alumno aprende son fundamentales para que se produzca un aprendizaje significativo, pero, para que esto ocurra, la evaluación debe pasar de la tradicional "evaluación del aprendizaje", observada en los discursos de los alumnos, a la "evaluación para el aprendizaje" (Torrance, 2007, p. 281), y los alumnos deben participar en el proceso de evaluación (Orsmond, Merry, \& Reiling, 2002).

Los cursos de formación del profesorado objeto de estudio tienen en cuenta una visión de la evaluación de los aprendizajes más asociada a una lógica sumativa (Boud \& Falchikov, 2006), que tiene como objetivo la clasificación y certificación, es decir, el propósito de la evaluación es proporcionar a los estudiantes un "certificado de aprovechamiento" (Thomas et al., 2019), destacando la importancia de las calificaciones solo para el uso de los resultados de la evaluación para las decisiones de selección, calificación, certificación o colocación (Van der Kleij et al., 2015). Sin embargo, los estudiantes entienden que la evaluación también desempeña un papel importante en el aprendizaje (Boud \& Falchikov, 2005; Lutovac $\&$ Flores, 2021a).

Una condición importante para que la evaluación apoye el aprendizaje es la implicación activa en el proceso de evaluación por parte de los propios alumnos (Black \& Wiliam, 1998). Como consecuencia, los estudiantes pueden contribuir activamente a la construcción de su propio conocimiento, lo que repercute en los resultados del aprendizaje (Sluijsmans, 2002), que se consigue gracias a una mayor participación de los estudiantes en las actividades del aula y en el propio proceso de evaluación.

La retroalimentación surgió en los discursos de los participantes como algo que no se muestra adecuado y oportuno (Gibbs, 1999), aunque la reconocen como una oportunidad de aprendizaje (Li \& Gao, 2016; Pope, 2001), es decir, está latente que estos estudiantes ven que la evaluación tiene que ver con la retroalimentación y la reflexión (Lutovac \& Flores, 2021a), aunque, en la práctica, no la viven como desearían. Para que el aprendizaje sea significativo, debe haber una evaluación guiada (López-Pastor \& Sicilia-Camacho, 2017). Otro aspecto importante es que la retroalimentación es una dimensión crítica en el desarrollo de una enseñanza de calidad y un aprendizaje eficaz en todos los ambientes educativos (Black \& William 1998; Carless et al., 2011; Tee $\&$ Ahmed, 2014). Sin embargo, sigue siendo un aspecto insatisfactorio de la experiencia de enseñanza y aprendizaje (Tee \& Ahmed, 2014) también destacado en este estudio.

Los estudiantes que serán futuros profesores, a diferencia de otros futuros profesionales, ya conocen el contexto en el que ejercerán su actividad: las escuelas y las aulas. Es una profesión que se ejercerá después de una prolongada exposición al contexto laboral, a través de la observación de sus profesores, que puede influir en su comprensión y práctica docente, tanto como futuros profesores como aprendices (Flores, 2010). Por lo tanto, es importante darles voz y espacio para la construcción del conocimiento profesional basado 
en sus experiencias como aprendices durante la formación inicial a través de la exposición y el análisis explícito de la pedagogía.

En este sentido, es fundamental considerar las concepciones y experiencias de aprendizaje y evaluación de los alumnos que serán futuros profesores, ya que pueden permitir una comprensión más amplia y profunda del proceso de ser profesor. Aunque las concepciones pueden variar en diferentes contextos, los resultados de los discursos de los estudiantes de magisterio proporcionan una comprensión de sus puntos de vista sobre la enseńanza, el aprendizaje y la evaluación y pueden ayudar a los profesores en formación a desarrollar sus habilidades de reflexión sobre estos conceptos (Wang et al, 2010; Lutovac \& Flores, 2021a). De ahí la importancia de comprender la dinámica de la enseñanza y el aprendizaje y la evaluación en los cursos de formación del profesorado en un contexto particular, incluida la reflexión sistemática y guiada sobre la experiencia como estudiantes de magisterio y su influencia en la forma de ver su práctica profesional y de imaginarse a sí mismos como profesores. Además, el análisis de la pedagogía en el contexto de la formación permite igualmente a los formadores de profesores reflexionar sobre sus prácticas y creencias de enseñanza y evaluación, así como sobre su eficacia en la formación de los estudiantes de magisterio.

\section{Referencias}

Allal, L. (2020). Assessment and the co-regulation of learning in the classroom. Assessment in Education: Principles, Policy \& Practice, 27(4), 332-349. https://doi.org/10.1080/0969594X. 2019.1609411

Allen, J.M., \& Wright, S.E. (2014). Integrating theory and practice in the pre-service teacher education practicum. Teachers and Teaching, 20(2), 136-151. https://doi.org/10.1080/13540602.2 013.848568

Almeida, L., Fernandes, E., Soares, A., Vasconcelos, R., \& Freitas, A. (2003). Envolvimento académico: Confronto de expectativas e comportamentos em universitários do $1 .^{\circ}$ ano. Revista Psicologia e Educação, 2(2), 57-70.
Almeida, L., \& Cruz, J. (2010). Transição e adaptação académica: reflexóes em torno dos alunos do $10^{\circ}$ ano da Universidade do Minho. In J. Silva et al. (orgs.), Ensino superior em mudança: tensôes e possibilidades: actas do Congresso Ibérico (pp. 429-440). CIEd.

Barr, R., \& Tagg, J. (1995). From teaching to learning - A new paradigm for undergraduate education. Change: The magazine of higher learning, 27(6), 12-26. https://doi.org/10.1080/000913 83.1995 .10544672

Bardin, L. (2013). Análise de Conteúdo. Edições 70. Black, P., \& D. Wiliam. (1998). Assessment and Classroom Learning. Assessment in Education. Principles, Policy \& Practice, 5(1), 7-74. https:// doi.org/10.1080/0969595980050102

Boud, D., \& Falchikov, N. (2005). Redesigning assessment for learning beyond higher education. Higher Education 28(special issue), 34-41.

Boud, D., \& Falchikov, N. (2006). Aligning assessment with long-term learning. Assessment \& evaluation in higher education, 31(4), 399-413. https://doi. org/10.1080/02602930600679050

Brown, G.T., \& Remesal, A. (2012). Prospective teachers' conceptions of assessment: A cross-cultural comparison. The Spanish Journal of Psychology, 15(1), 75-89. https://doi. org/10.5209/rev_SJOP.2012.v15.n1.37286

Brown, S. (2005). Assessment for Learning. Learning and Teaching in Higher Education, 1, 81-89. http://eprints.glos.ac.uk/id/eprint/3607

Bryman, A. (1988). Quantity and Quality in Social Research. Contemporary social research series, 18. Academic Division of Unwin Hyman, Ldt.

Carless, D. (2007). Learning-oriented assessment: conceptual bases and practical implications. Innovations in Education and Teaching International, 44(1), 57-66. https://doi. org/10.1080/ 14703290601081332

Carless, D.,Salter,D., Yang,M., \&Lam,J. (2011).Developing sustainable feedback practices. Studies in higher education, 36(4), 395-407. https:// doi.org/10.1080/03075071003642449 
Evans, L. (2010). Professionals or technicians? Teacher preparation programs and occupational understandings. Teachers and Teaching, 16(2), 183-205. https://doi.org/10.1080/13540600903478458

Flores, M.A. (2006). Being a Novice Teacher in Two Different Settings: Struggles, Continuities, and Discontinuities. Teachers College Record, 108(10), 2021-2052. https://doi.org/10.1111/ j.1467-9620.2006.00773.x

Flores, M.A. (2010). Algumas reflexóes em torno da formação inicial de professores. Educação, 33(3), 182-188. Pontifícia Universidade Católica do Rio Grande do Sul Porto Alegre, Brasil.

Flores, M.A. (2011). Curriculum of initial teacher education in Portugal: New contexts, old problems. Journal of Education for Teaching, 37(4), 461-470. https://doi.org/10.1080/026 07476.2011 .611015

Flores, M.A. (2016). Teacher Education Curriculum. In J. Loughran \& M. L. Hamilton (Eds.), International Handbook of Teacher Education (pp. 187-230). Springer Press.

Flores, M.A. (2017). Practice, theory, and research in initial teacher education: international perspectives. European Journal of Teacher Education, 40(3), 287-290. https://doi.org/10.1080/0261 9768.2017.1331518

Flores, M.A. (2019). Learning to be a teacher: mentoring, collaboration, and professional practice. European Journal of Teacher Education, 42(5), 535-538. https://doi.org/10.1080/02619768. 2019.1680207

Flores, M.A., Pereira, D., Fernandes, E., \& Coutinho, C. (2019). Conclusóes e Implicaçóes. In M. A. Flores (Eds.), Avaliação no Ensino Superior: Conceçóes e Práticas (pp. 229-254). De Facto Editores. Santo Tirso: Coleção Practicum - Ciências da Educação.

Freeman, M. (1995) Peer Assessment by Groups of Group Work. Assessment \& Evaluation in Higher Education, 20(3), 289-300. https://doi. org/10.1080/0260293950200305

Gibbs, G. (1999). Using Assessment Strategically to Change the Way Students Learn. In S. Brown \& A. Glasner (Eds.), Assessment Matters in Higher
Education: Choosing and Using Diverse Approaches (pp. 41-53). Open University Press.

Hammerness, K., van Tartwijk, J., \& Snoek, M. (2012). Teacher Preparation in the Netherlands: Shared Visions and Common Features. In L. Darling-Hammond \& A. Lieberman (Eds.), Teacher Education around the World. Changing Policies and Practices (pp. 44-65). Routledge.

Hughes, G. (2011) Towards a personal best: a case for introducing ipsative assessment in higher education. Studies in Higher Education, 36(3), 353-367. https://doi.org/10.1080/03075079. 2010.486859

Imig, D., Wiseman, D., \& Imig, S. (2011). Teacher Education in the United States of America. Journal of Education for Teaching, 37(4), 399-408. https://doi.org/10.1080/0260747. 2011.611006

Koscielniak, C. (2014). A consideration of the changing focus on the sustainable development in higher education in Poland. Journal of Cleaner Production, 62, 114-119. https://doi. org/10.1016/j.jclepro.2013.06.006

Korthagen, F., Loughran, J., \& Russell, T. (2006). Developing Fundamental Principles for Teacher Education Programs and Practices. Teaching and Teacher Education, 22, 1020-1041.

Krueger, R. \& Casey, M. (2009). Focus groups: A practical guide for applied research (4th Ed.). Sage.

Li, L., \& Gao, F. (2016). The effect of peer assessment on project performance of students at different learning levels. Assessment \& Evaluation in Higher Education, 41(6), 885-900. https://doi. org/10.1080/02602938.2015.1048185Loughran, J., Keast, S., \& Cooper, R. (2016). Pedagogical reasoning in teacher education. In International Handbook of Teacher Education, edited by J. Loughran and M. L. Hamilton (pp. 387-421). Springer Press.

López-Pastor, V., \& Sicilia-Camacho, A. (2017). Formative and shared assessment in higher education. Lessons learned and challenges for the future. Assessment \& Evaluation in Higher Education, 42(1), 77-97. https://doi.org/10.10 80/02602938.2015.1083535 
Lutovac, S., \& Flores, M. (2021a). Conceptions of assessment in pre-service teachers' narratives of students' failure. Cambridge Journal of Education. https://doi.org/10.1080/0305 764X.2021.1935736

Lutovac, S., \& Assunção Flores, M. (2021b). 'Those who fail should not be teachers': Pre-service Teachers' Understandings of Failure and Teacher Identity Development. Journal of Education for Teaching, 47(3), 379-394. https://doi. org/10.1080/02607476.2021.1891833Maschke, S., \& Stecher, L. (2016). Why Become a Teacher? Considerations on the Initial Study Phase in Teacher Training. Obrazovanie i samorazvitie - Education and Self-Development, 11(3), 36-44.

Mayer, D., Allard, A., Bates, R., Dixon, M., Doecke, B., Kline, J., \& Kostogriz, A. (2015). Studying the Effectiveness of Teacher Education: Final Report. Deakin University.

Mayer, D., Pecheone, R., \& Merino, N. (2012). Rethinking Teacher Education in Australia: The Teacher Quality Reforms. In L. Darling-Hammond and A. Lieberman (Eds.), Teacher Education around the World. Changing Policies and Practices (pp. 110-129). Routledge.

Miller, A., Imrie, B., \& Cox, K. (1998). Student Assessment in Higher Education: a handbook for assessing performance. Kogan Page.

Munthe, E., \& Rogne, M. (2015). Research Based Teacher Education. Teaching and Teacher Education, 46, 17-24. https://doi.org/10.1016/j.tate. 2014.10.006.

Myers, C., \& Myers, S. (2015). The use of learner-centered assessment practices in the United States: the influence of individual and institutional contexts. Studies in Higher Education, 40(10), 1904-1918. https://doi.org/10.1080/0307507 9.2014.914164

Nunes, S., \& Garcia, A. (2010). Estudantes do ensino superior: as relaçóes pessoais e interpessoais. Gestin, Revista da Escola Superior de Gestão, 195-203.
Orsmond, P., Merry, S., \& Reiling, K. (2002). The Use of Exemplars and Formative Feedback When Using Student Derived Marking Criteria in Peer and Self-Assessment. Assessment \& Evaluation in Higher Education, 2(4), 309-323. https:// doi.org/10.1080/0260293022000001337

Panadero, E., Jonsson, A., \& Strijbos, J.W. (2016). Scaffolding self-regulated learning through self-assessment and peer assessment: Guidelines for classroom implementation. In D. Laveault \& L. Allal (Eds.), Assessment for learning: Meeting the challenge of implementation (pp. 311-326). Springer International.

Pope, N. (2001). An Examination of the Use of Peer Rating for Formative Assessment in the Context of the Theory of Consumption Values. Assessment \& Evaluation in Higher Education, 26(3), 235-246. https://doi.org/10.1080/0260 2930120052396

Quivy, R., \& Campenhoudt, L. (2003). Manual de Investigação em Ciências Sociais. Ediciones Morata.

Ramon-Casas, M., Nuño, N., Pons, F., \& Cunillera, T. (2019). The different impact of a structured peer-assessment task in relation to university undergraduates' initial writing skills. Assessment \& Evaluation in Higher Education, 44(5), 653-663. https://doi.org/10.1080/02602938. 2018.1525337

Remesal, A. (2011). Primary and secondary teachers' conceptions of assessment: A qualitative study. Teaching and teacher education, 27(2), 472-482. https://doi.org/10.1016/j.tate.2010.09.017

Roldão, M.C. (2009). Estratégias de ensino. O saber e o agir do professor. Fundação Manuel Leão.

Rust, C. (2002). The impact of assessment on student learning: how can the research literature practically help to inform the development of departmental assessment strategies and learner-centred assessment practices? Active Learning in Higher Education, 3(2), 145-158. https://doi.org/10. 1177/1469787402003002004 
Seco, G., Pereira, A., Alves, S., Filipe, L., \& Duarte, A. (2011). Promoção de competências transversais no Ensino Superior. In Congreso Internacional Galego-Portugués de Psicopedagoxia, pp. 809-819.

Silverman, D. (2000). Doing qualitative research: a pratical handbook. Sage.

Sluijsmans, D. (2002). Establishing learning effects with integrated peer assessment tasks. The Higher Education Academy.

Tang, S. (2002). School-based learning in initial teacher education. Cardiff University.

Tang, S., Wong, A., \& Cheng, M. (2012). Professional Learning in Initial Teacher Education: Vision in the Constructivist Conception of Teaching and Learning. Journal of Education for Teaching, 38(4), 435-451. https://doi.org/10.1080/0260 7476.2012 .688549

Tang, S., Wong, A., \& Cheng, M. (2016). Examining professional learning and the preparation of professionally competent teachers in initial teacher education. Teachers and Teaching, 22(1), 54-69. https://doi.org/10.1080/13540602.201 5.1023028

Tang, S., Wong, A., Li, D., \& Cheng, M. (2019). Examining student teachers' engagement with the theory-practice link in initial teacher education. Journal of Education for Teaching, 45(2), 123-139. https://doi.org/10.1080/02607476.2 018.1548167

Tee, D.D., \& Ahmed, P.K. (2014). 360 degree feedback: An integrative framework for learning and assessment. Teaching in Higher Education, 19(6), 579-591. https://doi.org/10.1080/135 62517.2014 .901961

Thomas, D., Moore, R., Rundle, O., Emery, S., Greaves, R., te Riele, K., \& Kowaluk, A. (2019). Elaborating a framework for communicating assessment aims in higher education. Assessment \& Evaluation in Higher Education, 44(4), 546-564. https://doi.org/10.1080/02602938.2 018.1522615

Torrance, H. (2007). Assessment as Learning? How the Use of Explicit Learning Objectives, Assessment
Criteria and Feedback in Post-Secondary Education and Training Can Come to Dominate Learning. Assessment in Education, 14(3), 281-294. https://doi.org/10.1080/09695940701591867

Trindade, R. \& Cosme, A. (2010). Educar e aprender na escola: Questôes, desafios e respostas pedagógicas. Fundação Manuel Leitão.

Valeeva, R., \& Gafurov, I. (2017). Initial teacher education in Russia: connecting theory, practice, and research. European Journal of Teacher Education, 40(3), 342-360. https://doi.org/10. 1080/02619768.2017.1326480

Van Grieken, C. (2016). Formación docente y escuela: posibles articulaciones. Ciencia y Sociedad, 41(1), 29-43. https://doi.org/10.22206/cys. 2016. v41i1.pp029-043

Van der Kleij, F., Vermeulen, J., Schildkamp, K., \& Eggen, T. (2015). Integrating data-based decision making. Assessment for Learning and diagnostic testing in formative assessment. Assessment in Education: Principles, Policy \& Practice, 22(3), 324-343. https://doi.org/10.1 080/0969594X.2014.999024

Van Nuland, S. (2011). Teacher Education in Canada. Journal of Education for Teaching, 37(4), 409-421. https://doi.org/10.1080/02607476.2 011.611222

Wæge, K., \& Haugaløkken, O. (2013). Researchbased and hands-on practical teacher education: An attempt to combine the two. Journal of Education for Teaching, 39, 235-249. https:// doi.org/10.1080/02607476.2013.765195

Webber, K. (2012). The Use of Learner-Centered Assessment in US Colleges and Universities. Research in Higher Education, 53(2), 201-228. https://doi. org/10.1007/s11162-011-9245-0

Woolhouse, C., \& Cochrane, M. (2015). Educational Policy or Practice? Traversing the Conceptual Divide between Subject Knowledge, Pedagogy and Teacher Identity in England. European Journal of Teacher Education, 38(1), 87-101. https://doi. org/10.1080/02619768.2014.921154 